\title{
Medical 3D Integral Images Visualization in True Space
}

\author{
Mahmoud. G. Eljadid and Amar Aggoun
}

\begin{abstract}
D Integral Imaging (also referred to as 3D Holoscopic imaging) methodology uses the principle of "Fly's eye" and hence allows natural viewing of objects (i.e. fatigue free viewing); 3D-holoscopic technology produces images that are true optical models. This technology is based on physical principles with duplication of light fields. In this paper, a new method of visualization medical 3D integral images is proposed. Digital Imaging and Communications in Medicine data images (DICOM) taken from CT, MRI, PET and US images that produced by 3D-Doctor software to generate medical 3D integral images visualization of anatomy without glass in natural light. The method is mainly based on multiprocessor ray tracing system as renderer. The medical $3 \mathrm{D}$ content is captured in real time with the content viewed by multiple viewers independently of their position, without the needs of 3D eyewear. Experimental results show validation of the new algorithm and demonstrated that medical 3D integral images content can be displayed on commercially available multi-view auto-stereoscopic display. Medical 3D integral images content is parsed into multiprocessor ray tracing system, consequently, short time of medical 3D integral images movie of such pelvis scene is generated and displayed on PC screen, LCD and Holovizio display.
\end{abstract}

Index Terms-Computer graphics, medical 3D integral imaging processing \& visualization, animation, 3D TV.

\section{INTRODUCTION}

Medical applications 3D endoscopy and tomography for direct observation of the precise pathology of internal organs and non-invasive operations (Gastroscopes, Laparoscopes, Brain and Eye Surgery) and training. Creation of real-time 3D imaging system using traditional TV-technique for signal receiving and communication is now valid and certain application [1], [2].

The new method introduced 3D integral images as new technique based on multiprocessor ray tracer of representing DIACOM data images (CT/MRI/PET/US) to produce 3D integral images representations of anatomical structures that can be viewed pre and intra operatively (using augmented reality concept) without special eyewear in natural light. The technique developed proof of concept static display models Fig. 6. The proposed 3D integral images technology allows natural accommodation and convergence to accomplish stress-free viewing.

We have developed software tools based on multiprocessor ray tracer in the environment of Object-oriented in $\mathrm{C}++$ and $\mathrm{C}$ programming languages that

Manuscript received October 10, 2014; revised December 15, 2014.

Mahmoud G. Eljadid is with Tripoli University, Libya (e-mail: meljdid@hotmail.com).

Amar Aggoun is with Bedfordshire University, UK (e-mail: amar.aggoun@beds.ac.uk). allow 3D Integral images to be converted in the appropriate format supported by a commercial auto-stereoscopic display for viewing and interfacing in Fig. 5, Fig. 7 and Fig. 10.

\section{COMPUTER GENERATED 3D INTEGRAL IMAGES}

Integral imaging is attracting a lot of attention in recent year and has been regarded as strong candidate for next generation 3D TV [1]-[8]. Computer generation of integral imaging has been reported in several literatures [2]-[14]. A computer generated synthetic 3D integral image is presented as a two dimensional distribution of intensities termed a lenslet-encoded spatial distribution (LeSD), which is ordered directly by the parameters of a decoding array of micro lenses used to replay the three-dimensional synthetic image. When viewed, the image exhibits continuous parallax within a viewing zone dictated by the field angle of the array of micro-lenses. The replayed image is a volumetric optical model, which exists in space at a location independent of the viewing position. This occurs because, unlike stereoscopic techniques, which present planar perspective views to the viewer's eyes, each point within the volume of a 3D integral image is generated by the intersection of ray pencils projected by the individual micro-lenses. Due to the nature of the recording process of 3D integral image, many changes to the camera model used in standard computer generation software are carried out. To generate a unidirectional 3D integral image using a lenticular sheet, each lens acts like a cylindrical camera. A strip of pixels is associated with each lens forming a micro-image. Each cylindrical lens records a micro-image of the scene from a different angle as shown in the Fig. 1 and 5. For micro-lens arrays each lens acts like a square or a hexagonal camera depending on the structure of the lenses, as shown in Fig. 2. In the lateral cross section of the lenticular or the micro-lenses, a pinhole model is used. In the case of lenticular sheets, the pinhole forms a straight line parallel to the axis of the cylindrical lens in the vertical direction. For each pixel, a primary ray is spawned. The recording path of the primary ray draws a straight line going forward towards the image plane and backward away from the image plane. Similar primary rays of neighbouring lenses are spawned to similar directions parallel to each other. Therefore highly correlated micro-images are produced which, is a property of $3 \mathrm{D}$ integral images.

The structure of the lenses and the camera model in the in 3D Integral images computer graphics affects the way primary rays are spawned as well as the spatial coherence among them.

The camera model used for each micro-lens is the pinhole approximation, where each micro-lens acts like a separate camera. The result is a set multiple cameras. Each of them records a micro-image of the virtual scene from a different 
angle see Fig. 3 and 4. Primary rays pass through the centre of the micro-lens and the image plane. The scene image straddles the micro-lens array. Therefore there are two recording directions, in front and behind the micro-lens array. The specific characteristics of 3D integral images, allows us to deal with each cylindrical lens separate from the others, and to measure the number of pixels behind each lens, focal length and the image width. All these parameters including the number of lenslets in the virtual cylindrical array are selected on the basis of the characteristics of the display device.

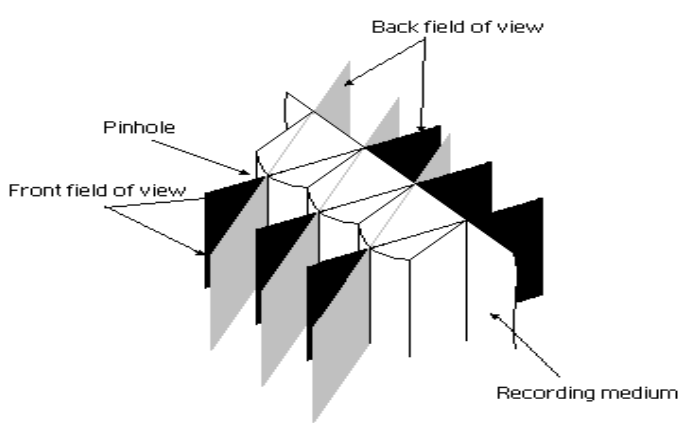

Fig. 1. Lenticular sheet model in integral ray tracer.

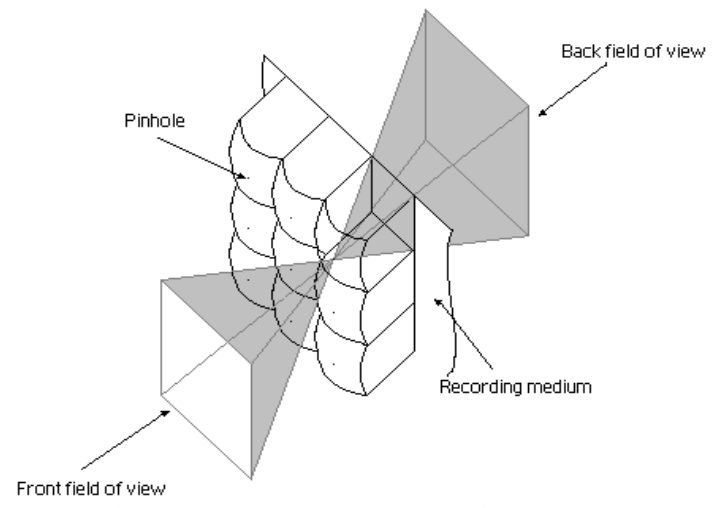

Fig. 2. Micro-lens array in integral ray tracing.

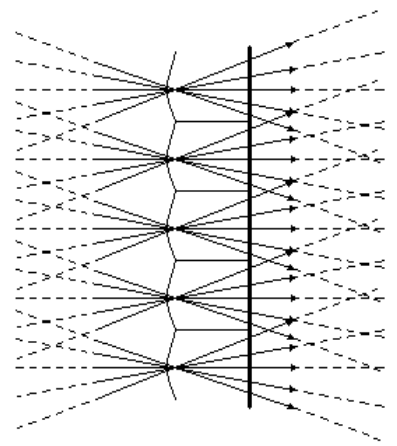

Fig. 3. Camera model in 3D integral images for computer graphics.

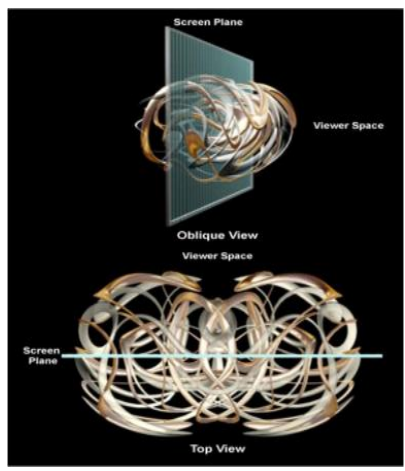

Fig. 4. Screen plane showing the position of integral image.

The pixels intensity values of the micro-image for each lenslet are read, saved, and then mapped to pixels locations on the screen so that all the vertical slots are displayed at the same time forming the $3 \mathrm{D}$ integral image. The location of the vertical elemental image on the computer screen is identical to the location of the corresponding lenslet in the virtual lenses array Fig. 4.

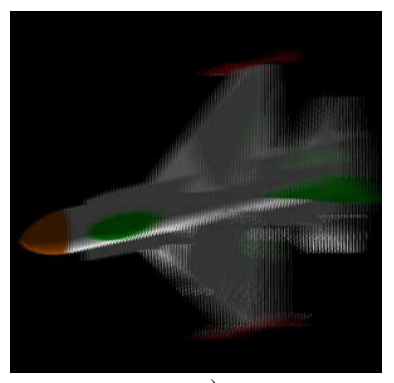

a)

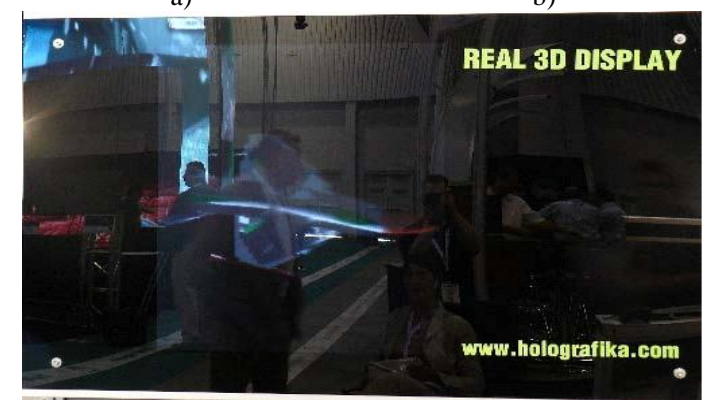

c)

Fig. 5. a) 3D integral images (holoscopic) frame of falcon scene [1]. b) 3D holoscopic display using and LCD panel with a lenticular [1]. c) Display of 3D holoscopic image on the holografika system [3].

\section{Medical 3D InTEgRal IMAGES CONTENT PARSER PLUG-IN FOR MULTIPROCESSOR RAY TRACING SYSTEM}

The medical data obtained through CT, MRI and PTE is exported in Digital Imaging and Communications in medicine format. A segmentation process is then applied to manually highlight the regions of interest in each slice, using commercially available software such 3D-Doctor [9], Fig. 6. The question raised here, how to accept/import medical 3D model file format and generate a 3D integral images, new plug-in software is developed to allow interfacing the computer generation of 3D Integral images graphics software with CT, MRI, PET and US Fig. 8.

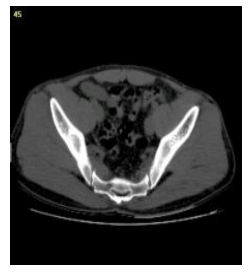

a) Original CT image.

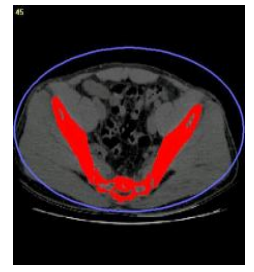

b) Segmentation

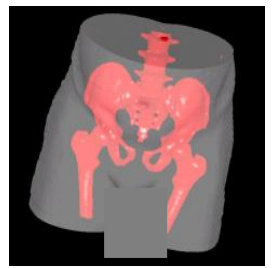

c) 3D mesh model created. Fig. 6. Digital imaging and communications in medicine (DICOM) data from CT [9].
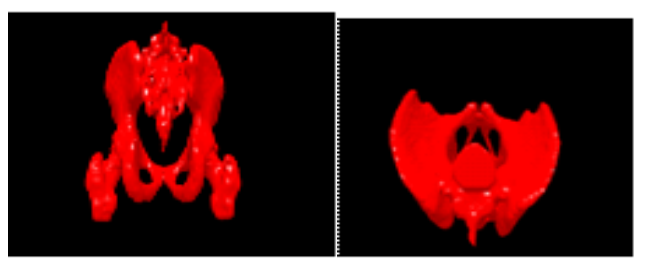

Fig. 7. 3D pelvis model display by 3D-Doctor viewer. software. 


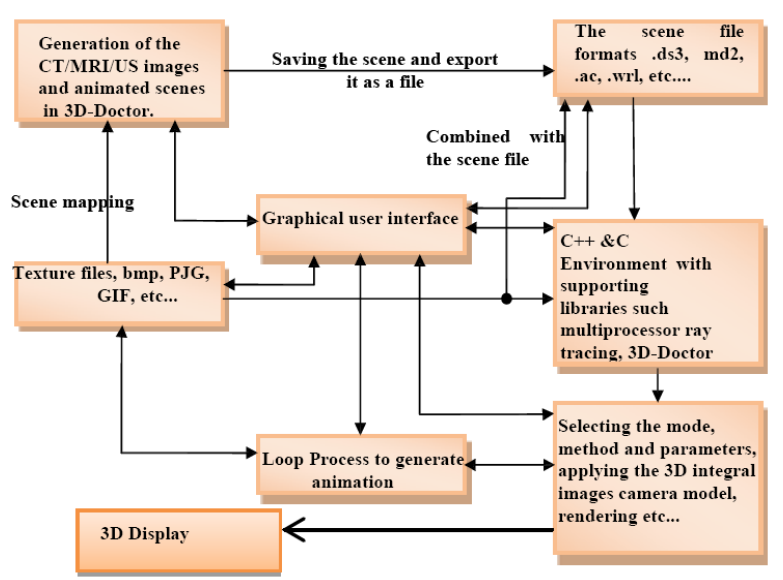

Fig. 8. Flowchart of the medical plug-in tool.

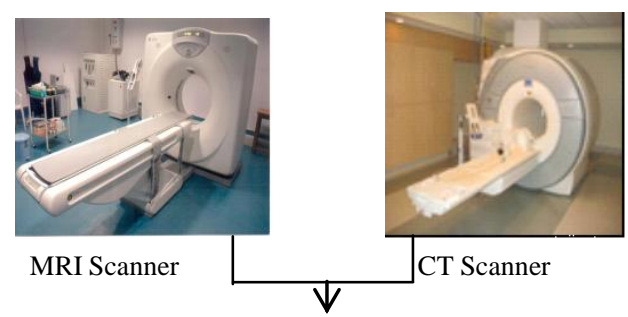

(CT/MRI/PET/US) IMAGES

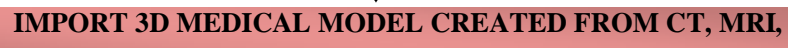
PTE and US IMAGES

3D UNIDIRECTIONAL CAMERA PARAMTERS

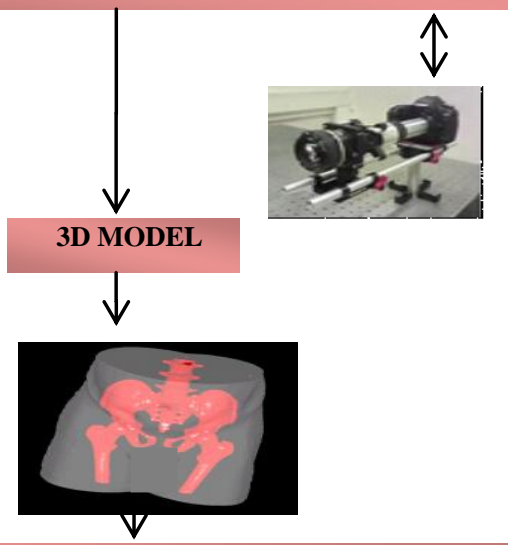

ENVIRONMENT OF OBJECT-ORIENTED PROGRAMMING IN C++ AND C LANGUGUE BASED ON MULTIPROCESSOR RAY TRACER

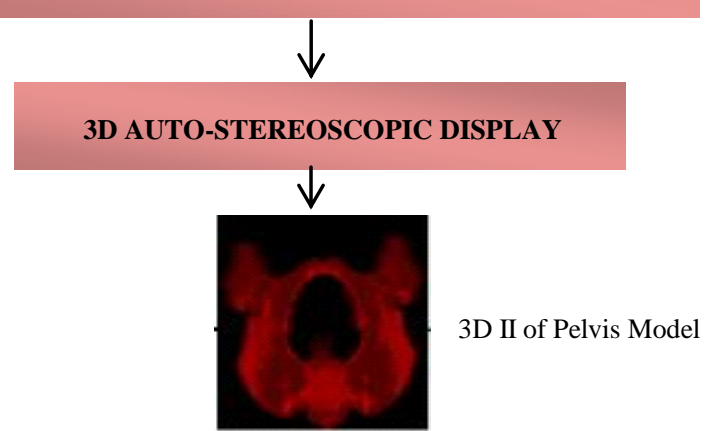

Fig. 9. Flow chart of the medical 3D integral images visualization generator.

The 3D integral multiprocessor ray tracing (3D IRT) software has been modified in order to incorporate medical

3D integral imaging parser [15]. That composed of two main phases that deal with the medical 3D scene description file format. The first phase is to build/develop a routine of 3D MIIP in order to analyse a continuous flow of text-based input. It breaks the input into its constituent parts in order to read, handle and parse the $3 \mathrm{D}$ integral images camera model parameters as a stream of tokens Fig. 8 and 9 and (see Table I). The second phase is to develop another routine to deal with the rest of medical 3D model file format such as materials, objects, etc.

Parsing processes is consisted of three main phases:

1) Reading medical $3 \mathrm{D}$ model content file.

2) Converting medical $3 \mathrm{D}$ model to multiprocessor ray tracing.

3) Rendered the scene description model on Multiprocessor ray tracing engine.

\section{EXPERIMENTS AND RESULTS}

The results are extremely satisfactory and for the first time it is proved that medical 3D integral images (holoscopic) content can be generated through multiprocessor / parallel ray tracer and displayed on commercially available multi-view auto-stereoscopic display. In this paper a unidirectional integral images camera model is adopted (see Table I). An example of a 3D Pelvis rendered using the multiprocessor integral imaging ray tracer is shown in Fig. 10. 612 frames have been generated with image size $1024 \times 768$, and 9 pixels behind each cylindrical lens.

TABLE I: 3D UNIDIRECTIONAL CAMERA PARAMETERS

\begin{tabular}{lll}
\hline \hline Parameters & \multicolumn{2}{c}{ Lenticular sheet } \\
\hline Resolution & 1024768 & {$[$ Pixel] } \\
Lens Pitch & 2.116667 & {$[\mathrm{~mm}]$} \\
Lens Pixels & 9 & {$[$ Pixel] } \\
Aperture Distance & 10.0 & [mm] \\
Number of lenses & 113 & [Lens] \\
Focal Length & 6.8 & [mm] \\
Ray Depth & 2 & [Integer] \\
\hline \hline
\end{tabular}

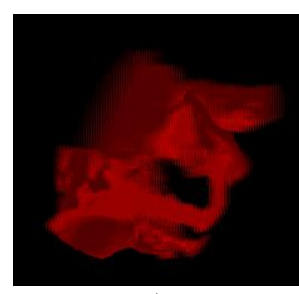

a)

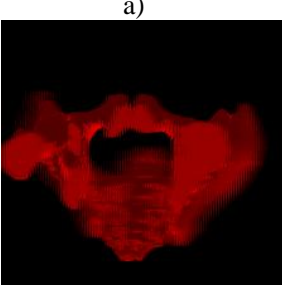

c)

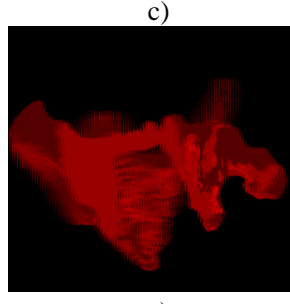

e)

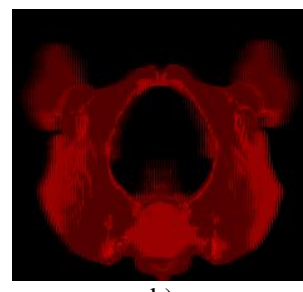

b)

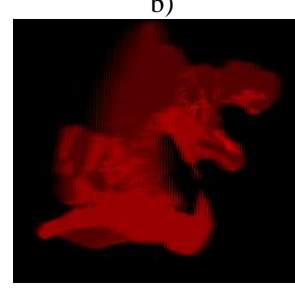

d)

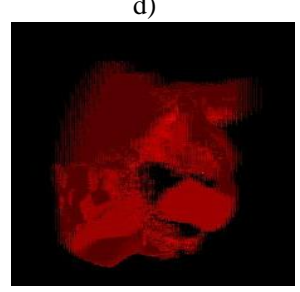

f) 


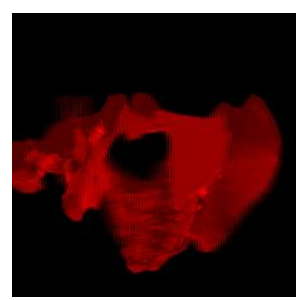

g)

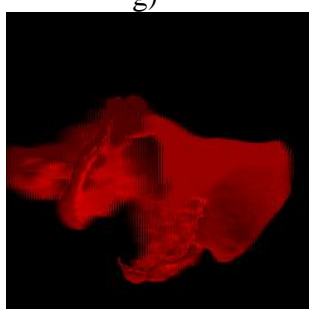

i)

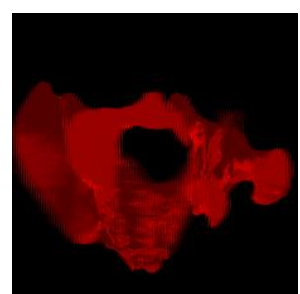

h)

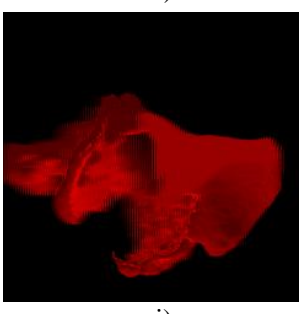

j)
Fig. 10. a)-j) Computer generated a sequence of medical 3D integral images frames.

\section{CONCLUSION}

This paper presents a new multiprocessor ray tracing system software tool to allow visualization of medical 3D integral images. In conclusion, we are able to display 3D integral images relevant to minimally invasive urology. The results have demonstrated that medical $3 \mathrm{D}$ integral images content can be displayed on commercially available multi-view auto-stereoscopic display.

\section{REFERENCE}

[1] M. G. Eljdid, A. Aggoun, and O. H. Youssef, "Computer generated content for 3D TV," in Proc. 3D TV Conf., Kos Island, Greece, May 2007.

[2] J. K. Makanjuola et al., "3D-holoscopic imaging: A new dimension to enhance imaging in minimally invasive therapy in urology oncology," Journal of Enduorology, vol. 27, issue 5, May 2, 2013.

[3] Contract no: IST-7-248420-STREP. (April 20, 2012). Program FP7-ICT-2009-4. Intermediate Dissemination Report. [Online]. Available: http://www.3dvivant.eu/

[4] A. Aggoun, E. Tsekleves, D. Zarpalas, P. Daras, A. Dimou, L. Soares, and P. Nunes, "Immersive 3D holoscopic system," IEEE Multimedia Magazine, Special Issue on 3D Imaging Techniques and Multimedia Applications, vol. 20, issue 1, pp. 28-37, Jan.-Mar. 2013.

[5] M. G. Eljdid, A. Aggoun, and O. H. Youssef, "Enhanced techniques 3D integral images video computer generated," presented at the
International Conference on Computing Technology and Information Management, Dubai, UAE, April, 2014.

[6] G. Lippmann, "La photographie integrale," ComtesRendus, Academie des Sciences, vol. 146, pp. 446-451, 1908.

[7] M. G. Eljdid, "3D content computer generation for volumetric displays," Ph.D. dissertation, Dept. Electronic and Computer Eng., Brunel University., London, 2007.

[8] A. Aggoun: "3D holoscopic imaging technology for real-time volume processing and display," High Quality Visual Experience Signals and Communication Technology, 2010, pp. 411-428.

[9] 3D-Doctor. [Online]. Available: http://www.3d-doctor.com/

[10] O. A. Fatah et al., "Generating stereoscopic 3D from holoscopic 3D," presented at 3D TV Conf., Aberdeen, United Kingdom, October, 2013,

[11] J. Hong, J.-H. Park, S. Jung, and B. Lee, "A depth-enhanced integral imaging by use of optical path control," Opt. Lett., vol. 29, no. 15, pp. 1790-1792, 2004

[12] S.-W. Min et al., "Three-dimensional display system based On computer-generated integral imaging," Stereoscopic Display and Virtual Reality Systems VIII Proc. the SPIE, vol. 4297, pp. 187-195, June 2001

[13] F. Okano et al., "Real-time pickup method for a three-dimensional image based on integral photography," Apply Optical, vol. 36, pp. 1598-1604, 1997.

[14] B. Javidi and F. Okano, Three-Dimensional Television, Video, and Display Technologies, New York: Springer, 2002.

[15] A. V. Aho, M. S. Lan et al., Compilers Principles, Techniques \& Tools, New York: Pearson Addison, 2006, ch. 2-4.

Mahmoud G. Eljadid is a lecturer in information and communication technologies at Tripoli University, Libya. His research interests include computer generation and live capture of 3D integral images using Multiprocessor ray tracing system, 3D integral images content, 3D medical visualization, 3D video coding and animation, computer vision systems, and real-time digital image/video processing, 3D software plug-in tools, acceleration techniques for computer generation 3D integral images (real-time), 3D integral images games, analysis and design of engineering information systems. Eljadid has a $\mathrm{PhD}$ in electronic and computer engineering from Brunel University, West London, UK.

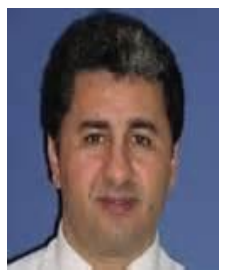

Amar Aggoun is a professor in information and communication technologies at Bedfordshire University, UK. His research interests include light-field imaging systems, computer generation and live capture of 3D integral images, depth measurement and volumetric data reconstruction, 3D medical visualization, 3D video coding, computer vision systems, and real-time digital image/video processing. Aggoun has a $\mathrm{PhD}$ in electronic engineering from the University of Nottingham, UK. 